\title{
Analysis of pendulums coupled by torsional springs for en- ergy harvesting
}

\author{
$P$ V Malaji ${ }^{1, *}$, Suresh Doddi ${ }^{1, * *}$, Michael I. Friswell ${ }^{2, * * *}$, and Sondipon Adhikari ${ }^{2, * * * *}$ \\ ${ }^{1}$ BLDEA,s V P Dr. P G Halakatti College of Engineering and Technology, Vijayapur, Karnataka, India \\ ${ }^{2}$ Swansea University, Swansea, UK
}

\begin{abstract}
Harvesting energy from ambient sources has been a recent topic of interest. A typical linear harvester is effective only near resonance, limiting its frequency bandwidth. In order to increase the efficiency and bandwidth of harvesters, various strategies have been proposed. Using multiple harvesters in a single device can harvest enough power over wider frequency band. In the present work, the effect of torsional coupling of the harvesters for low frequency vibration energy harvesting is investigated. Two pendulums with electromagnetic induction as the energy conversion mechanism is proposed. The performance of the device is studied theoretically and numerically. Cubic polynomials are used to model the pendulum nonlinearity. Fundamental harmonic oscillation are assumed to obtain the analytical solution. The effect of torsional coupling and pendulum length on the power harvested are reported.
\end{abstract}

\section{Introduction}

The concept of harvesting energy from ambient sources has been the focus of the energy harvesting community. Ambient energy sources exist in various forms such as solar, wind, tidal, mechanical vibrations and many more $[1,2]$. Energy harvesting converts this ambient energy into electrical energy, which can be used for low-power devices. Out of the available sources, converting mechanical vibrations into electrical energy is the latest trend in research. Vibration can be converted into electrical energy via electrostatic, electromagnetic and piezoelectric transduction [2].

The conventional linear harvesters produce maximum power at resonance and are hence called resonant harvesters [3]. This puts limitation on the power output of the harvester at wider frequencies. Various strategies have been proposed to increase the bandwidth of harvesters [4]. Frequency tuning, multiple harvester and nonlinear harvesters are most studied approaches. The method of frequency tuning of the harvester adjusts the frequency of the harvester to match with the source [5], so that the operating frequency range is increased. However, studies have pointed out that tuning the harvesters to resonating conditions is often infeasible [6]. Nonlinearity of the harvesters can be used to extract power over wide frequency bands [7-9]. Magnetopiezoelastic [7] and inverted cantilever beam [9] are the most widely studied nonlinear harvesters.

\footnotetext{
*e-mail: pradeepmalaji@bldeacet.ac.in

**e-mail: suresh2010.uvce@gmail.com

***e-mail: M.I.Friswell@swansea.ac.uk

****e-mail: s.adhikari@swansea.ac.uk
} 
Use of multiple or multi-modal harvesters [10] has received the most attention and this approach is considered in the present study. While a close eigenmode provides a broadband application with lower power output, a large frequency gap between the modes makes these harvesters ineffective for harvesting power over a continuous frequency band [11]. Mechanical coupling of these multiple harvesters enhances both the magnitude and bandwidth of power [12]. Multiple harvesters with mechanical coupling have been explored in our previous studies [13,14]. Most of the work related to coupled harvesters were carried out on the basis of numerical studies. The present work considers Harmonic Balance Method (HBM) for analysis and compares with numerical results.

In this article, two torsionally coupled nonlinear pendulums for broadband electromagnetic energy harvesting is studied. Two pendulums are connected with a torsional springs (see Fig. 1). The pendulum lengths are mistuned to obtain increased bandwidth. Effect of coupling these two mistuned harvesters is analyzed using the HBM and a numerical study.

This paper is organised as follows. Section 2 describes the mathematical model and the electromechanical equations that describe the system. Section 3 compares the HBM and numerical results. The salient features emerging from this study are summarized in section 4 .

\section{Harvester model}

The mathematical model of the harvesting system with torsionally coupled pendulums is reported in this section. Figure. 1 shows the pendulums of length $l_{1}$ and $l_{2}$ are coupled by torsional spring of stiffness $k$.

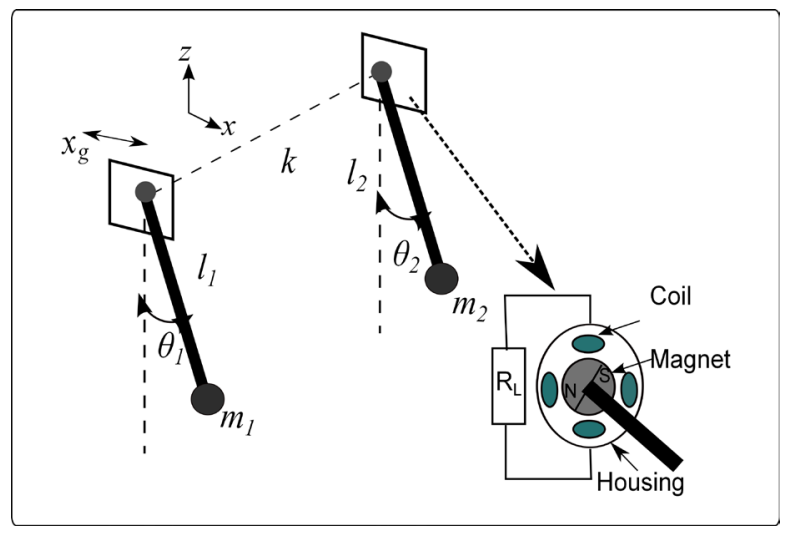

Figure 1: Schematic representation of coupled harvester model.

Equation of motion for pendulums are given as;

$$
\begin{aligned}
& m_{1} l_{1}^{2} \ddot{\theta}_{1}+c l_{1}^{2} \dot{\theta}_{1}+m_{1} g l_{1} \sin \theta_{1}+k\left(\theta_{1}-\theta_{2}\right)=-m_{1} l_{1} \ddot{x}_{g} \cos \theta_{1} \\
& m_{2} l_{2}^{2} \ddot{\theta}_{2}+c l_{2}^{2} \dot{\theta}_{2}+m_{2} g l_{2} \sin \theta_{2}+k\left(\theta_{2}-\theta_{1}\right)=-m_{2} l_{2} \ddot{x}_{g} \cos \theta_{2}
\end{aligned}
$$

Where, damping coefficient is sum of mechanical and electrical damping $c=c_{m}+c_{e}$.

The voltage induced by electromagnetic induction due to the electromagnetic flux $B$, length of coil $L$ and magnet length $l_{m}$ is given as

$$
\begin{aligned}
& v_{1}=B L l_{m} \dot{\theta_{1}} \\
& v_{2}=B L l_{m} \dot{\theta_{2}}
\end{aligned}
$$


To ease simulations and understanding of the system, the following dimensionless parameters are introduced.

$$
\begin{array}{ccccc}
\mu_{i}=\frac{m_{i}}{m_{1}}, & \alpha_{i}=\frac{l_{i}}{l_{1}}, & \omega_{1}=\sqrt{\frac{g}{l_{1}}}, & \gamma=\frac{c}{m_{1} \omega_{1}}, & \beta=\frac{k}{m_{1} l_{1}^{2} \omega_{1}^{2}}, \\
f=\frac{X_{g}}{l_{1}}, & \omega=\frac{\omega_{f}}{\omega_{1}}, & \tau=\omega_{1} t, & x_{g}=X_{g} \cos \omega_{f} t, & i=1,2 .
\end{array}
$$

Substituting the non-dimensional parameters from Eq. (3) into Eqs. (1) and (2) leads to nondimensional equations, which are given as,

$$
\begin{gathered}
\mu_{1} \alpha_{1}^{2} \ddot{\theta_{1}}+\gamma \dot{\theta_{1}}+\mu_{1} \alpha_{1} \sin \theta_{1}+\beta\left(\theta_{1}-\theta_{2}\right)+=\mu_{1} \alpha_{1} f \cos \omega t \cos \theta_{1} \\
\mu_{2} \alpha_{2}^{2} \ddot{\theta}_{2}+\gamma \dot{\theta_{2}}+\mu_{2} \alpha_{2} \sin \theta_{2}+\beta\left(\theta_{2}-\theta_{1}\right)+=\mu_{2} \alpha_{2} f \cos \omega t \cos \theta_{2} \\
v_{1}=\dot{\theta_{1}} \\
v_{2}=\dot{\theta_{2}}
\end{gathered}
$$

where $\omega_{1}$ is the uncoupled natural frequency of first pendulum. $\mu_{i}$ is the ratio of mass of each pendulum w.r.t. mass of the first pendulum $\left(m_{1}\right)$. Similarly, $\alpha_{i}$ denotes length ratio of each pendulum w.r.t. the first pendulum length. Hence, $\mu_{1}=1$ and $\alpha_{1}=1$ always. A parameter $\beta$ represents the coupling between the pendulums.

$\cos \theta_{i}$ and $\cos \theta_{i}$ terms are expanded up to cubic terms and fundamental harmonic oscillations are assumed.

$$
\begin{gathered}
\theta_{i}=a_{i}(t) \sin (\omega t)+b_{i}(t) \cos (\omega t) \\
\theta_{2}=a_{2}(t) \sin (\omega t)+b_{2}(t) \cos (\omega t) \\
v_{1}=A_{i}(t) \sin (\omega t)+B_{i}(t) \cos (\omega t) \\
v_{2}=A_{2}(t) \sin (\omega t)+B_{2}(t) \cos (\omega t)
\end{gathered}
$$

Substituting Eqs. (5) into expanded form of Eqs. (4) and balancing the terms multiplied by $\sin \omega t$ and $\cos \omega t$, the following steady state equations are obtained:

$$
\begin{aligned}
& -\mu_{1} \alpha_{1}^{2} a_{1} \omega^{2}-\beta a_{2}-\frac{1}{8} \mu_{1} \alpha_{1} a_{1} b_{1}^{2}-\frac{1}{8} \mu_{1} \alpha_{1} a_{1}^{3}-\gamma b_{l} \omega \\
& +\mu_{1} \alpha_{1} a_{1}+\frac{1}{4} \mu_{1} \alpha_{1} f \omega^{2} a_{1} b_{1}+\beta a_{1}=0 \\
& -\mu_{1} \alpha_{1}^{2} b_{l} \omega^{2}-\beta b_{2}+\gamma a_{l} \omega+\beta b_{1}-\frac{1}{8} \mu_{l} \alpha_{l} a_{l}^{2} b_{1}+\frac{1}{8} \mu_{l} \alpha_{1} f \omega^{2} a_{l} \\
& +\mu_{1} \alpha_{1} b_{1}-\frac{1}{8} \mu_{1} \alpha_{1} b_{1}{ }^{3}+\frac{3}{8} \mu_{1} \alpha_{1} f \omega^{2} b_{1}^{2}-\mu_{1} \alpha_{1} f \omega^{2}=0 \\
& A_{1}+a_{1} \omega=0 \\
& B_{1}+b_{1} \omega=0-\mu_{2} \alpha_{2}^{2} a_{2} \omega^{2}-\beta a_{2}-\frac{1}{8} \mu_{2} \alpha_{2} a_{2} b_{2}^{2}-\frac{1}{8} \mu_{2} \alpha_{2} a_{2}^{3}-\gamma b_{2} \omega \\
& +\mu_{2} \alpha_{2} a_{2}+\frac{1}{4} \mu_{2} \alpha_{2} f \omega^{2} a_{2} b_{2}+\beta a_{2}=0 \\
& -\mu_{2} \alpha_{2}^{2} b_{1} \omega^{2}-\beta b_{2}+\gamma a_{1} \omega+\beta b_{1}-\frac{1}{8} \mu_{2} \alpha_{2} a_{2}^{2} b_{1}+\frac{1}{8} \mu_{2} \alpha_{2} f \omega^{2} a_{2} \\
& +\mu_{2} \alpha_{2} b_{2}-\frac{1}{8} \mu_{2} \alpha_{2} b_{2}{ }^{3}+\frac{3}{8} \mu_{2} \alpha_{2} f \omega^{2} b_{2}{ }^{2}-\mu_{2} \alpha_{2} f \omega^{2}=0 \\
& A_{2}+a_{2} \omega=0 \\
& B_{2}+b_{2} \omega=0
\end{aligned}
$$


The voltage amplitude is given by;

$$
\begin{aligned}
& V_{1}=\sqrt{A_{1}^{2}+B_{1}^{2}} \\
& V_{2}=\sqrt{A_{2}^{2}+B_{2}^{2}}
\end{aligned}
$$

The next section compares results from the HBM and numerical simulation obtained from Eq. (4) using the Runge-Kutta method. The dimensionless parameters used are $\alpha_{1}=1$, $\alpha_{2}=1.1, \gamma=0.025, f=0.02$ and $\mu_{1}=\mu_{2}=1$.

\section{Results and discussion}

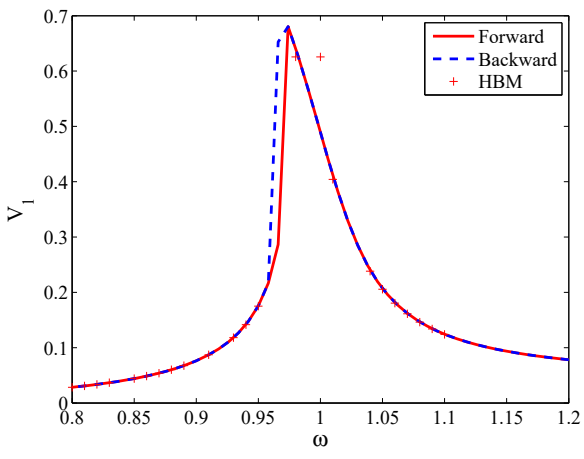

(a)

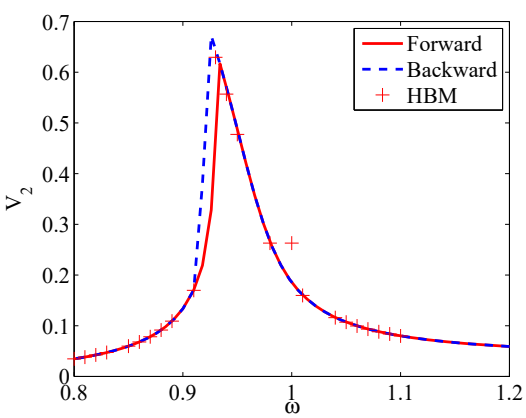

(b)

Figure 2: Voltage curves for two pendulum harvester at $\beta=0$. (a) Pendulum-1 and (b) Pendulum-2.

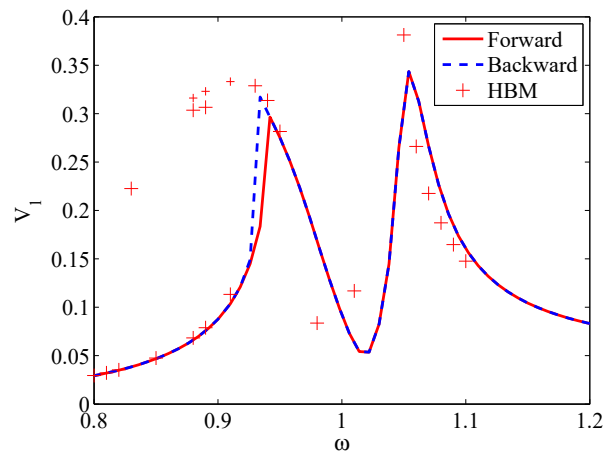

(a)

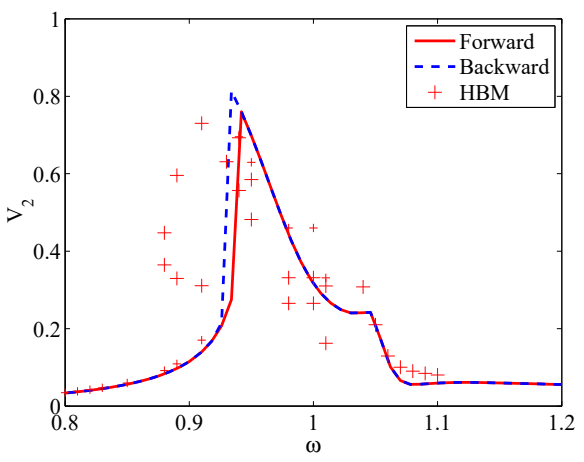

(b)

Figure 3: Voltage curves for two pendulum harvester at $\beta=0.08$. (a) Pendulum-1 and (b) Pendulum-2. 


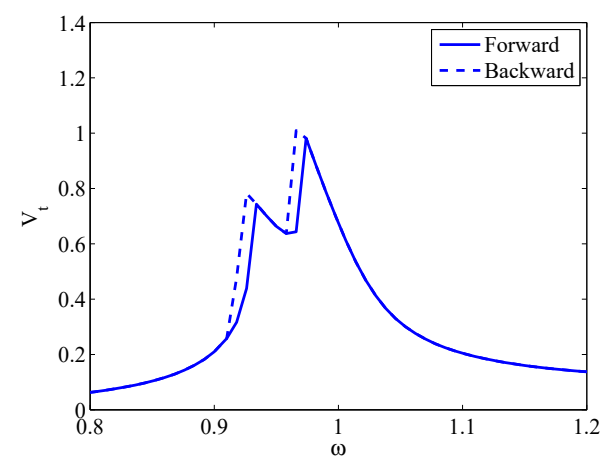

(a)

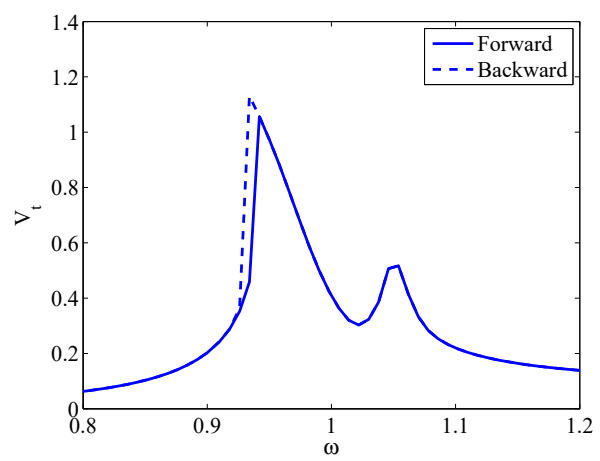

(b)

Figure 4: Total voltage curves for two pendulum harvester. (a) $\beta=0$ and (b) $\beta=0.08$.

To investigate the system, the numerical results and Harmonic Balance results are compared. Figure 2 shows voltage output for forward and backward sweep excitation for uncoupled harvesters $(\beta=0)$. It can be seen that both pendulums can harvest the peak voltage at different frequencies due to the difference in the pendulum lengths. A spring softening effect can also be observed. The HBM and numerical results match well.

The effect of coupling on the voltage response can be observed from Fig. 3. Two peaks of voltage with reduced amplitude for Pendulum-1 can be observed due to the coupling which indicates multifrequency harvesting. An increase in both the voltage amplitude and the frequency bandwidth at the voltage amplitude of 0.2 is observed. Qualitative similarity in the HBM and numerical results are observed however quantitative differences exist. This may be due to number of terms assumed for the HBM.

Total voltage harvested from the pendulums is obtained by adding individual voltages, the numerical results for total voltage are shown in Fig. 4. At a voltage amplitude of 0.2 the coupled harvester exhibits higher bandwidth $(\omega=0.92-1.08)$ compared to the uncoupled pendulums $(\omega=0.92-1.02)$.

\section{Conclusion}

In this paper, harmonic balance and numerical methods are applied to obtain the frequency response functions of the coupled pendulum harvester to compare the relative accuracy of the two methods in characterizing the response of nonlinear harvesters. The HBM and numerical results closely match when the pendulums are not coupled compared to the coupled pendulums. More detailed analysis with different parameters needs to be carried out in the future.

\section{References}

[1] C. Williams, R. Yates, Proceedings of the International Solid-State Sensors and Actuators Conference - TRANSDUCERS '95 1, 8 (1995)

[2] J.A. Paradiso, T. Starner, IEEE Pervasive Computing 4, 18 (2005)

[3] S.F. Ali, M.I. Friswell, S. Adhikari, Smart Materials and Structures 19, 1 (2010)

[4] R. Ramlan, Ph.D. thesis (2009) 
[5] V.R. Challa, M.G. Prasad, Y. Shi, F.T. Fisher, Smart Materials and Structures 17, 015035 (2008)

[6] S. Roundy, Y. Zhang, Toward self-tuning adaptive vibration based micro-generators, in Proceedings of SPIE (2005), Vol. 5649, pp. 373-384

[7] A. Erturk, J. Hoffmann, D.J. Inman, Applied Physics Letters 94, 1 (2009)

[8] S.F. Ali, S. Adhikari, M.I. Friswell, S. Narayanan, J. Applied Physics 109, 1 (2011)

[9] M.I. Friswell, S.F. Ali, O. Bilgen, S. Adhikari, A.W. Lees, G. Litak, Journal of Intelligent Material Systems and Structures 23, 1505 (2012)

[10] M. Rajarathinam, S. Ali, Energy Conversion and Management 155, 10 (2018)

[11] I. Sari, T. Balkan, H. Kulah, Sensors and Actuators, A: Physical 145-146, 405 (2008)

[12] P. Malaji, S.F. Ali, Sensors \& Actuators A: Physical 255, 1 (2017)

[13] P.V. Malaji, S.F. Ali, The European Physical Journal Special Topics 224, 2823 (2015)

[14] P. Malaji, S.F. Ali, Mechanical Systems and Signal Processing 108, 304 (2018) 